\title{
Spawning Microhabitat Selection in Wild-Caught Delta Smelt Hypomesus transpacificus under Laboratory Conditions
}

\author{
Joan C. Lindberg ${ }^{1} \cdot$ Yi-Jiun J. Tsai ${ }^{1} \cdot$ Brittany D. Kammerer $^{2} \cdot$ Bradd Baskerville-Bridges $^{1} \cdot$ Tien-Chieh Hung $^{1}$ (D)
}

Received: 19 August 2019 /Revised: 8 November 2019 / Accepted: 17 November 2019 / Published online: 17 December 2019

(C) The Author(s) 2019

\begin{abstract}
Very little is understood about the spawning habitat of endangered delta smelt Hypomesus transpacificus, which hinders ongoing recovery efforts such as wetland habitat restoration and spawning habitat augmentation. To address this, the spawning response of wild-caught $H$. transpacificus to different substrates and water velocities was examined across three experiments. In experiment 1, spawning response to dead wood, pebble, natural and artificial Schoenoplectus acutus, empty tray, and tank floor at water velocities of 1.4 and $8.8 \mathrm{~cm} / \mathrm{s}$ was tested. Egg deposition on pebble at $8.8 \mathrm{~cm} / \mathrm{s}$ velocity $(78.1 \%$ of 7778 total eggs) was significantly greater than that on all other substrate-velocity combinations. In experiment 2 , spawning response to natural S. acutus, dead wood, sand, pebble, cobble, and empty tray at velocities of 8.7 and $15.4 \mathrm{~cm} / \mathrm{s}$ was tested. Egg deposition on pebble at $15.4 \mathrm{~cm} / \mathrm{s}(61.5 \%$ of 36171 total eggs) was significantly greater than that on all substrate-velocity combinations except for sand at $15.4 \mathrm{~cm} / \mathrm{s}$. Sand at $15.4 \mathrm{~cm} / \mathrm{s}(22.3 \%)$ contained significantly more eggs than all combinations except for pebble at 8.7 and $15.6 \mathrm{~cm} / \mathrm{s}$. In experiment 3, egg attachment to natural S. acutus, dead wood, sand, pebble, cobble, and empty tray when exposed to $14.6 \mathrm{~cm} / \mathrm{s}$ velocity was tested. Egg loss on sand was significantly greater than that on empty tray and pebble, indicating that egg deposition on sand may have been underestimated in experiment 2. Together, these results indicate that H. transpacificus selects pebble and sand at higher water velocities for spawning under certain laboratory conditions. These findings are important, as they provide insight into the potential natural habitats that may be used for spawning and thereby inform ongoing habitat restoration efforts.
\end{abstract}

Keywords Delta smelt $\cdot$ Spawning preference $\cdot$ Substrate $\cdot$ Water velocity $\cdot$ Microhabitat selection

\section{Introduction}

Delta smelt Hypomesus transpacificus is small pelagic fish native to the upper estuary of the San Francisco Estuary and Sacramento-San Joaquin Delta in northern California. Due to

Communicated by Mark S. Peterson

Electronic supplementary material The online version of this article (https://doi.org/10.1007/s12237-019-00672-5) contains supplementary material, which is available to authorized users.

Tien-Chieh Hung

thung@ucdavis.edu

1 Department of Biological and Agricultural Engineering, Fish Conservation and Culture Laboratory, University of California, Davis, Davis, CA 95616, USA

2 Department of Anatomy, Physiology, and Cell Biology, School of Veterinary Medicine, University of California, Davis, Davis, CA 95616, USA their low population abundance, $H$. transpacificus is listed as endangered by the state (Department of Fish and Game 2011) and threatened by the federal government (US Fish and Wildlife Service 1993). Hypomesus transpacificus is thought to be an important indicator of the health of native fish species in the delta (Moyle et al. 2018), and its designation as endangered has led to significant conservation efforts, such as wetland habitat restoration and limiting freshwater diversion (Moyle et al. 2018). However, very little is understood regarding spawning in $H$. transpacificus, which hinders ongoing recovery measures. For example, the Delta Smelt Resiliency Strategy (California Natural Resources Agency 2016) is an action plan drafted by state and federal agencies to improve conditions for $H$. transpacificus that specifically calls for spawning habitat augmentation. But without a fundamental understanding of H. transpacificus spawning habitat, it is unlikely that this action can be addressed adequately and effectively. Thus, to inform such recovery efforts, spawning microhabitat selection in H. transpacificus was examined here. 
Primarily an annual fish, $H$. transpacificus, is thought to generally move from the low salinity rearing habitat (e.g., Suisun Bay) to spawn upstream in fresher waters of the delta periphery (Radtke 1966; Sommer et al. 2011). In terms of spawning habitat, it has been hypothesized that $H$. transpacificus may spawn on sandy beaches (Bennett 2005; Moyle et al. 2016), submerged tree branches (Wang 2010), larger rock rubble (Bennett 2005), or in open water above sandy or rocky substrates (Wang 2010). However, neither spawning behavior nor eggs have been observed in the wild (Bennett 2005; Interagency Ecological Program 2015; Moyle et al. 2016), so the true spawning habitat of H. transpacificus is unknown.

In this study, the spawning response of wild-caught $H$. transpacificus to different substrates and water velocities was examined across three experiments under laboratory conditions where egg deposition and attachment could be quantified. In experiment 1 , egg deposition on dead wood, pebble, natural and artificial Schoenoplectus acutus, empty tray, and tank floor at water velocities of 1.4 and $8.8 \mathrm{~cm} / \mathrm{s}$ was tested ( $N=2$ tanks, March 1-April 13, 2002). In experiment 2, egg deposition on natural $S$. acutus, dead wood, sand, pebble, cobble, and empty tray at water velocities of 8.7 and $15.4 \mathrm{~cm} / \mathrm{s}$ was tested $(N=4$ tanks, February 20-March 29 , 2004). In experiment 3 , to determine whether there was a bias in egg attachment to certain substrates, egg attachment to natural $S$. acutus, sand, pebble, cobble, dead wood, and empty tray (substrates tested in experiment 2) when exposed to $14.6 \mathrm{~cm} / \mathrm{s}$ velocity for 3 days was tested ( $N=8$ trays, 2004).

\section{Methods}

All experiments were conducted at the Fish Conservation and Culture Laboratory of University of California, Davis, near Byron California, USA. Fish Conservation and Culture Laboratory staff captured and supplied all $\mathrm{H}$. transpacificus (Lindberg et al. 2013).

\section{Experiments 1 and 2}

\section{Experimental Design}

Wild-caught $H$. transpacificus adults were stocked into fiberglass tanks $\left(37.21 \mathrm{~m}^{3}, 6.1 \mathrm{~m}\right.$ diameter $\times 1.63 \mathrm{~m}$ depth) at 100 fish per tank and an approximately 50:50 sex ratio. Tanks were located outdoors and exposed to natural photoperiod and temperatures (range $=9.5-26.1{ }^{\circ} \mathrm{C}$ ). Fish were allowed to acclimate for 2 weeks prior to both experiments with spawning substrates in place. Fish were fed a commercial diet (Lindberg et al. 2013) two times a day ad libitum.

Within each holding tank, water $(76.2 \mathrm{~cm}$ height) flowed around the tank in one direction and exited through a central drain. Water velocity was highest near the tank wall and decreased toward the central drain in a gradient. For experiment 1 , unidirectional inflow was created by six spigots $(1.9 \mathrm{~cm}$ PVC elbow fittings) spaced equidistant apart along the rim of each tank (Fig. S1). Substrates were arranged in two concentric rings at mean $( \pm$ SE) water velocities of $1.4 \pm 0.04 \mathrm{~cm} / \mathrm{s}$ and $8.8 \pm 0.4 \mathrm{~cm} / \mathrm{s}$ within each of two tanks. Each velocity tested contained two replicates (single trays) of each substrate. In experiment 2 , inflow was provided by two stacked spray bars (122 cm long, $7.6 \mathrm{~cm}$ diameter PVC) anchored to the floor and angled such that flow hit the wall before circulating around the tank (Fig. S2). Substrates were arranged in two concentric rings at mean $( \pm \mathrm{SE})$ water velocities of $8.7 \pm$ $0.7 \mathrm{~cm} / \mathrm{s}$ and $15.8 \pm 1.0 \mathrm{~cm} / \mathrm{s}$ within each of four tanks. Substrate patch size was increased to three trays of the same substrate adjacent to one another. One replicate (tray-triplet) of each substrate type was placed within each velocity tested.

Within each velocity tested in experiment 1 , egg deposition on artificial $S$. acutus did not differ from that on natural S. acutus, and egg deposition on tank floor did not differ from that on empty tray (Tukey's multiple comparison test, $P>0.05$ ). As a result, artificial $S$. acutus and tank floor substrates were replaced with sand and cobble in experiment 2 , allowing for more natural substrates to be tested within given space and time constraints.

For both experiments, substrates were placed equidistant from one another within each water velocity and offset between velocities (Figs. S1, S2). Substrate order was randomized within each water velocity and tank, except for natural S. acutus in experiment 2. Due to its height and volume, natural $S$. acutus in experiment 2 reduced water velocity when placed upstream of other substrates, necessitating that it be placed in last position (Fig. S2). Substrate order remained the same for the duration of each experiment.

\section{Water Velocity}

Water velocity was measured three times per week using an electromagnetic sensor (model 2000 Marsh-McBirney, Inc., MD, USA) placed at each of the two corners of the leading edge of the substrate trays. Water velocity was calculated as the mean of these measurements. The water velocities tested were chosen based on what could be achieved in the large tanks and agreed with Swanson et al. (1998), in which H. transpacificus could swim in velocities up to $\sim 27 \mathrm{~cm} / \mathrm{s}$, but began to demonstrate swimming failure, decreased endurance, and an altered swimming gait at about $10-15 \mathrm{~cm} / \mathrm{s}$.

\section{Substrate Construction}

All substrates except for tank floor were arranged on plastic trays $(30 \mathrm{~cm} \mathrm{~W} \times 40 \mathrm{~cm} \mathrm{~L} \times 2 \mathrm{~cm} \mathrm{H})$. Buoyant trays (artificial and natural $S$. acutus, empty tray, and deadwood) were 
clamped to anchor trays glued to the tank floor. Tank floor substrate was a marked area of the tank floor equal to tray size. Natural S. acutus, dead wood, sand, pebble, and cobble were collected from the shores of the lower Sacramento River and canals around the Fish Conservation and Culture Laboratory property.

Artificial and natural S. acutus substrates consisted of 20 stalks per tray (61 cm height). Artificial $S$. acutus was constructed of clear acrylic tubing (12 $\mathrm{mm}$ outer diameter) that was spray painted green and leached. Stalks were oriented vertically within the water column and secured to trays in a randomly-selected configuration that was the same across all natural and artificial S. acutus substrates.

For dead wood substrate, two to three dead wood pieces were cut to fit lengthwise on trays (3-8 cm diameter, 30 $35 \mathrm{~cm}$ height), and then fastened to trays using stainless steel wood screws. Sand substrate consisted of medium-to-coarsegrained sand that was sieved through a 1000-mesh screen (most particles were 250-500 $\mu \mathrm{m}$ ), and then glued to trays using clear silicone adhesive. Pebbles (mean size $=$ $2.9 \mathrm{~cm} \mathrm{~L} \times 1.8 \mathrm{~cm} \mathrm{~W} \times 1.3 \mathrm{~cm} \mathrm{H}$ ) were spread evenly to fill the tray. Cobble substrate with low vertical profile (ca. $20 \mathrm{~cm}$ $\mathrm{L} \times 25 \mathrm{~cm} \mathrm{~W} \times 7 \mathrm{~cm} \mathrm{H}$ ) were placed on trays.

\section{Egg Quantification}

Eggs were sampled three times per week by draining the tank to $\sim 30 \mathrm{~cm}$ height, retrieving trays, and counting eggs. Fish were accustomed to tank draining, as it was part of their daily care prior to these experiments. Eggs were naturally adhesive, and only eggs attached to substrates were counted.

Different techniques were used to quantify egg deposition. For natural and artificial S. acutus, cobble, dead wood, and empty tray substrates, the number of eggs attached to the surface of each substrate was counted. For sand, eggs were dislodged with a strong water stream, sieved through a $500 \mu \mathrm{m}$ Nitex ${ }^{\circledR}$ screen, and then counted. For pebble, the sand protocol for egg removal was used if less than 30 eggs were observed on the surface of the substrate. If 30 or more eggs were observed, then chemical de-adhesion was used instead, as it allowed for eggs adhered to underlying pebbles to be retrieved without damage. For chemical de-adhesion, pebbles were soaked in dilute liquid bleach $(0.8 \%)$ for $3 \mathrm{~min}$, agitated for an additional minute, soaked in freshwater, and then sprayed with water to dislodge loosened eggs. Collected water was then sieved and resulting eggs were counted. Pebbles were discarded after chemical treatment. For the tank floor substrate, excessively turbid water conditions necessitated that trained personnel use their fingers to find eggs. Eggs were then scraped off the floor and collected. After quantifying egg deposition on all substrates, tanks were lightly brushed down and drained to $\sim 20 \mathrm{~cm}$ to rid the tanks of extra debris before being refilled.

\section{Data Analyses}

Spawning microhabitat selection was quantified as the number of eggs deposited onto each substrate exposed to a given water velocity. For both experiments, a generalized linear model with a negative binomial distribution and a $\log \operatorname{link}$ function best fit the data compared to linear regression models and generalized linear models with poisson, zero-inflated poisson, and zero-inflated negative binomial distributions (Zuur et al. 2009). No obvious overdispersion was observed in simulated residuals of the models used (DHARMa package in R).

Generalized linear models for both experiments included substrate, velocity, and the interaction between the two as fixed factors. Tank and date were included as fixed factors to account for variation between tanks and through time, respectively. For the response variable, the total number of eggs counted for each substrate within each velocity for each tank was used. For experiment 1, egg counts were pooled across replicates within a given velocity for each tank and sampling date. For experiment 2, egg counts were pooled across all three trays of a tray-triplet (a single replicate) within a given velocity for each tank and sampling date. Based on backward selection and analysis of deviance tests (Zuur et al. 2009), all terms were included in our final model for both experiments.

Significant substrate by velocity interactions in both experiments were further analyzed using Tukey's multiple comparison tests. These comparisons were determined a priori based on graphical examination of the data; pebble at $8.8 \mathrm{~cm} / \mathrm{s}$ velocity was compared with all other substrate-velocity combinations in experiment 1 , and both pebble and sand at $15.4 \mathrm{~cm} / \mathrm{s}$ velocity were compared with all other substrate-velocity combinations in experiment 2 .

\section{Experiment 3}

\section{Experimental Design}

Eggs and sperm were manually expressed from wild-caught $H$. transpacificus, mixed, and then applied to substrates submerged in $2 \mathrm{~cm}$ of water. Eggs became adhesive once exposed to water. The number of eggs attached to each substrate was quantified. Substrates were then exposed to a mean $( \pm \mathrm{SE})$ water velocity of $14.6 \pm 1.0 \mathrm{~cm} / \mathrm{s}$ within a single tank for 3 days, the approximate number of days between samplings in experiments 1 and 2. Following exposure, the number of attached eggs remaining on each substrate was quantified. Tank plumbing was configured as described in experiment 2 .

\section{Substrate Construction}

Small samples $(7 \mathrm{~cm} \times 10 \mathrm{~cm})$ of each substrate type were glued to plastic trays $(N=8)$. Each tray contained one sample of each substrate type placed in a $3 \times 2$ configuration in 
random order. All samples except for natural $S$. acutus were prepared using the same methods described for experiments 1 and 2. In experiment 3, natural $S$. acutus was glued flat onto trays. Trays were rinsed in water for $24 \mathrm{~h}$ before use, and then clamped to anchor trays within the tank.

\section{Egg Quantification}

On all substrate types, the number of eggs in a $2.3 \mathrm{~cm} \times 2.3 \mathrm{~cm}$ area was visually counted before and after exposure. Subsampled areas were chosen to be representative of overall egg distribution, have eggs in a single layer, and be easily resampled. Egg counts were not extrapolated to the total substrate area, as the subsampled area was not randomly chosen and may have been biased against clumped egg distribution.

\section{Data Analyses}

The number of eggs that remained attached to each substrate type after exposure to $14.6 \mathrm{~cm} / \mathrm{s}$ water velocity was examined using a linear regression. For this model, the number of attached eggs remaining after exposure was the response variable, substrate type and tray were fixed explanatory variables, and the number of eggs attached prior to exposure was a covariate. Tray was not a significant factor $(P>0.05)$, so it was dropped from the model. In the final model, the substrate type by covariate interaction was significant $(P<0.05)$, so pairwise comparisons with Tukey's $P$ value adjustments were used to examine the differences in slopes between substrate types (emmeans package in R). Simple linear regressions were also used to examine the relationship between the number of attached eggs present before and after exposure for each substrate type separately.

In nine samples, the number of eggs counted after exposure was greater than that counted before exposure (up to 3 additional eggs), which was attributed to human error. These samples were adjusted such that the number of eggs counted after exposure was equal to the number of eggs counted before. Statistical analyses with this adjustment showed similar results to analyses excluding these samples. Only analyses with adjusted samples are reported below.

Data for all experiments are provided in Tables S1, S2, S3 . R (R Core Team 2018) and RStudio (RStudio Team 2016) were used for all statistical analyses. $\mathrm{R}$ code can be provided upon request to the corresponding author.

\section{Results}

\section{Experiment 1}

For experiment 1, 7778 eggs were counted across all substrates and velocities across the entire sampling period and both tanks. Substrates exposed to $8.8 \mathrm{~cm} / \mathrm{s}$ water velocity (7102 eggs, $91.3 \%$ of all eggs counted) contained more eggs than those exposed to $1.4 \mathrm{~cm} / \mathrm{s}$ (676 eggs, $8.7 \%)$. The substrate-velocity containing the greatest number of eggs was pebble in $8.8 \mathrm{~cm} / \mathrm{s}$ water velocity (6077 eggs, 78.1\%), followed by dead wood at $8.8 \mathrm{~cm} / \mathrm{s}(376 \mathrm{eggs}, 4.8 \%)$ and pebble at $1.4 \mathrm{~cm} / \mathrm{s}$ (355 eggs, $4.6 \%)$.

When these data were examined statistically, date (likelihood ratio test (LR), $\chi^{2}=41.89, \mathrm{df}=1, P<0.001$ ), tank (LR, $\left.\chi^{2}=4.09, \mathrm{df}=1, P=0.04\right)$, and the interaction between substrate and velocity $\left(\mathrm{LR}, \chi^{2}=50.80, \mathrm{df}=5, P<0.001\right)$ were all significant factors. In particular, the number of eggs found on pebble exposed to $8.8 \mathrm{~cm} / \mathrm{s}$ velocity was significantly higher than that found on all other substrate-velocity combinations (Tukey's, $P<0.05$ ). The back-transformed estimated marginal means $( \pm$ SE) of egg counts for all substrates and velocities in both experiments are shown in Fig. 1.

The number of eggs counted across all tanks, substrates, and velocities generally decreased over time. When date was removed from the generalized linear model, all significant results described above remained the same.

\section{Experiment 2}

For experiment 2, 36171 eggs were counted across all substrates and velocities across the entire sampling period and all four tanks. Substrates exposed to $15.4 \mathrm{~cm} / \mathrm{s}$ water velocity (30987 eggs, $85.7 \%$ of all eggs counted) contained more eggs than those exposed to $8.7 \mathrm{~cm} / \mathrm{s}$ (5184 eggs, $14.3 \%)$. The substrate velocities containing the greatest number of eggs were pebble and sand substrates exposed to $15.4 \mathrm{~cm} / \mathrm{s}$ velocity (22232 eggs, $61.5 \%$ and 8050 eggs, $22.3 \%$, respectively), followed by pebble at $8.7 \mathrm{~cm} / \mathrm{s}(3391$ eggs, $9.4 \%)$ and sand at $8.7 \mathrm{~cm} / \mathrm{s}(970$ eggs, $2.7 \%)$.

When these data were examined statistically, date (LR, $\left.\chi^{2}=25.89, \mathrm{df}=1, P<0.001\right), \operatorname{tank}\left(\mathrm{LR}, \chi^{2}=75.01, \mathrm{df}=3\right.$, $P<0.001$ ), and the interaction between substrate and velocity (LR, $\left.\chi^{2}=59.05, \mathrm{df}=5, P<0.001\right)$ were all significant factors. In particular, the number of eggs found on pebble substrate exposed to $15.4 \mathrm{~cm} / \mathrm{s}$ velocity did not differ from that on sand at $15.4 \mathrm{~cm} / \mathrm{s}$ (Tukey's, $P>0.05$ ), but did contain significantly more eggs than all other substrate-velocity combinations (Tukey's, $P<0.05$ ). Sand substrate exposed to $15.4 \mathrm{~cm} / \mathrm{s}$ velocity did not differ from pebble exposed to either 8.7 or $15.6 \mathrm{~cm} / \mathrm{s}$ velocities (Tukey's, $P>0.05$ ), but did contain significantly more eggs than all other substrate-velocity combinations (Tukey's, $P<0.05$ ).

The number of eggs counted across all tanks, substrates, and velocities varied through time and showed no obvious patterns. The significant results described above remained the same both when date was treated as a random factor and when date was removed from the model. 
Fig. 1 Graph showing backtransformed estimated marginal means $( \pm \mathrm{SE})$ of eggs counted on each substrate type and within each water velocity for experiments 1 and 2. Estimated marginal means $( \pm \mathrm{SE})$ were based on generalized linear models with negative binomial distributions and $\log$ link functions. In experiment 1 (top panel, $N=2$ tanks), pebble substrate exposed to $8.8 \mathrm{~cm} / \mathrm{s}$ velocity contained significantly more eggs than all other substrate-velocity combinations (Tukey's, $P<0.05$ ). In experiment 2 (bottom panel, $N=4$ tanks), pebble exposed to $15.4 \mathrm{~cm} / \mathrm{s}$ velocity contained significantly more eggs than all substrate-velocity combinations (Tukey's, $P<0.05$ ) except for sand at $15.4 \mathrm{~cm} / \mathrm{s}$ (Tukey's, $P>0.05)$. Sand at $15.4 \mathrm{~cm} / \mathrm{s}$ contained significantly more eggs than all other substrate-velocity combinations (Tukey's, $P<0.05$ ) except for pebble at either 8.7 or $15.6 \mathrm{~cm} / \mathrm{s}$ (Tukey's, $P>0.05$ )
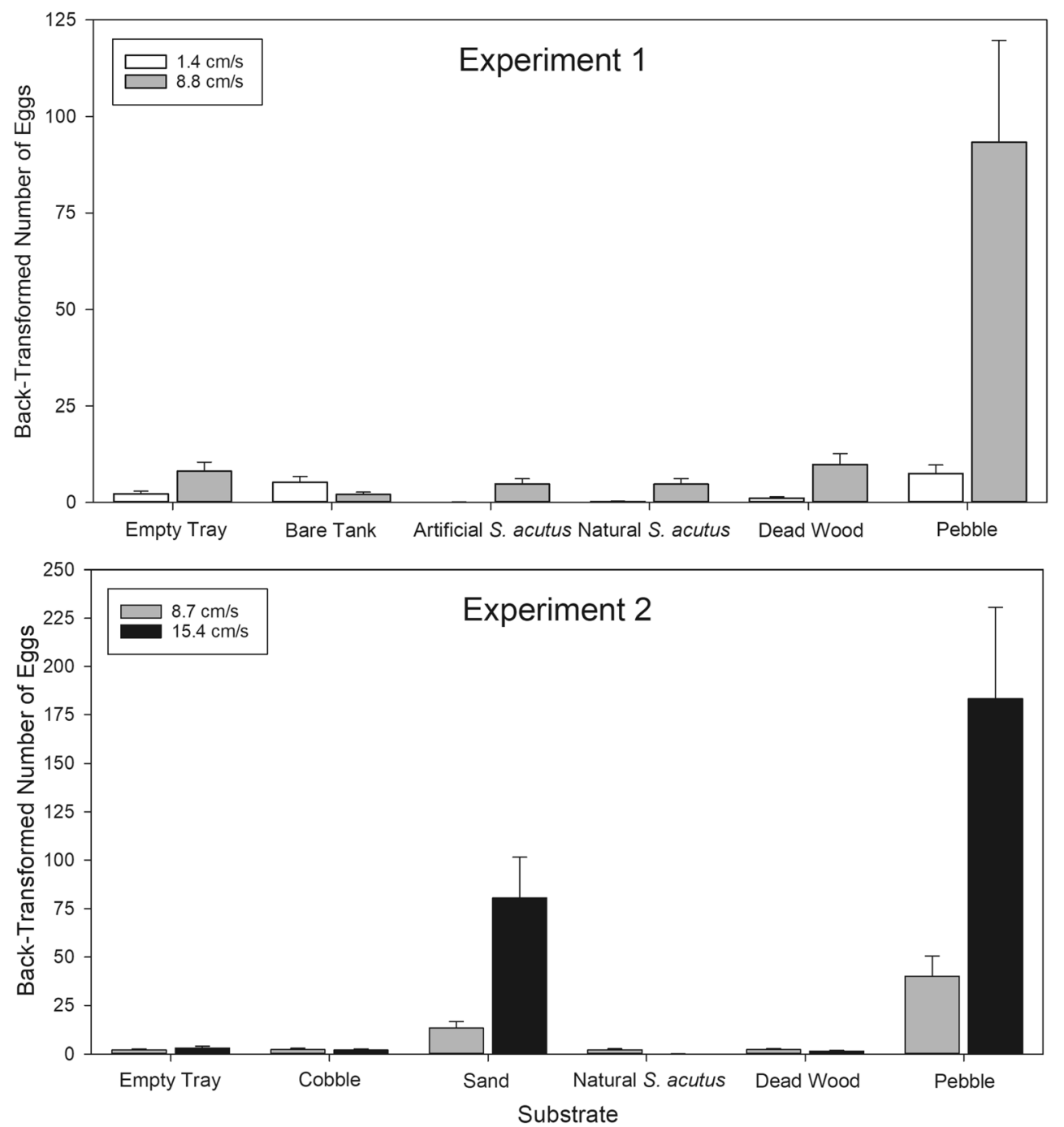

\section{Experiment 3}

Prior to exposure, 955 eggs were counted across all substrate types. After exposure, 774 eggs remained attached. Within each substrate, $86.4 \%$ of eggs remained on cobble, $68.9 \%$ remained on dead wood, $95.9 \%$ remained on empty tray, $85.3 \%$ remained on pebble, $59.4 \%$ remained on sand, and $88.6 \%$ remained on natural S. acutus.

The linear regression model showed a significant interaction between the number of eggs present before exposure and substrate type $\left(F_{5,36}=6.48, P=0.0002\right.$, Fig. 2$)$. Specifically, egg loss on sand was significantly higher than that on empty tray and pebble (Tukey's, $P<0.05$ ). Also, egg loss on dead wood was significantly higher than that on empty tray (Tukey's, $P<0.05$ ). All other comparisons were not significant (Tukey's, $P>0.05$ ).

When examined individually, the linear relationship between the number of eggs present before and after exposure was significant for all substrates (Adj $R^{2}>0.43, P<0.05$ ) except for sand (Adj $R^{2}=-0.07, F_{1,6}=0.54, P=0.49$ ). Notably, the relationship for empty tray was extremely strong (Adj $\left.R^{2}=0.98, F_{1,6}=408.0, P<0.001\right)$.

\section{Discussion}

Understanding spawning habitat selection in H. transpacificus is vital to implementing effective conservation measures for species recovery. To address this need, we examined $H$. transpacificus spawning response to different substrates and water velocities across three experiments. In experiments 1 and 2, we found a significant interaction between substrate and velocity, which indicates that both factors are important to $H$. transpacificus spawning. Specifically, within the range of substrates and velocities tested, pebble and sand exposed to higher water velocities contained the greatest number of eggs, and may therefore be preferred over S. acutus, dead wood, cobble, artificial substrates, and lower water velocities. Notably, we believe that our quantification of egg deposition on sand in experiment 2 may be underestimated. In 


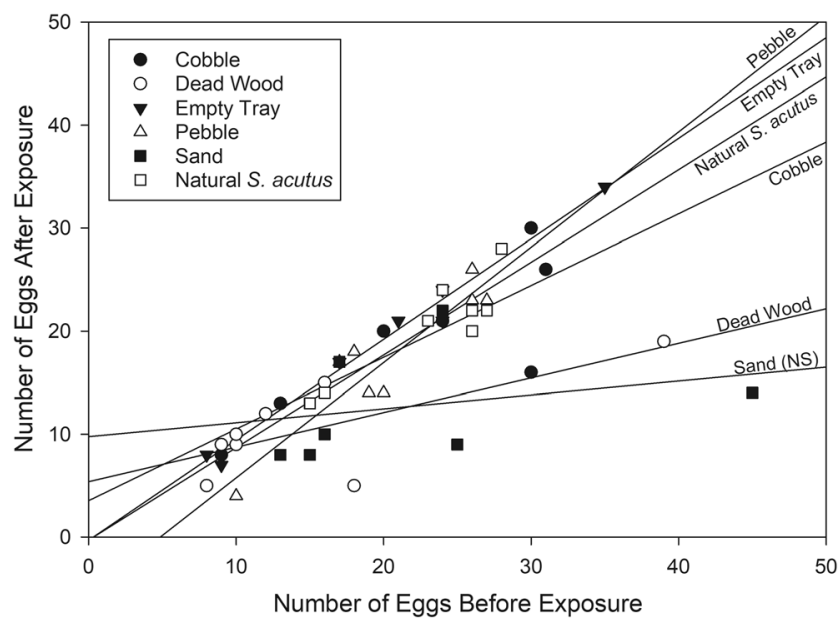

Fig. 2 Graph showing the linear relationship between the number of eggs attached before and after exposure to $14.6 \mathrm{~cm} / \mathrm{s}$ water velocity for each substrate type in experiment 3 . Egg loss on sand was significantly higher than that on empty tray and pebble (Tukey's, $P<0.05$ ), and egg loss on dead wood was significantly higher than that on empty tray (Tukey's, $P<0.05$ ). There were strong (Adj. $\left.R^{2}>0.43\right)$ and significant $(P<0.05)$ relationships for all substrates except for sand $(P>0.05$, non-significance denoted by NS)

experiment 3 , we found that egg loss on sand was significantly greater than that on empty tray or pebble. There was also no significant linear relationship between the number of eggs present on sand before and after exposure to $14.6 \mathrm{~cm} / \mathrm{s}$ water velocity. This indicates that many eggs deposited on sand were lost after exposure, regardless of the number initially present. Thus, $H$. transpacificus spawning response to sand may be stronger than what is shown here.

Our finding that $H$. transpacificus deposited the most eggs on sand and pebble is perhaps not surprising, given that the species is thought to spawn in freshwater sloughs of the delta (Bennett 2005), where sand beds and areas of pebble mixed with sand and small clam shells are relatively abundant (e.g., Sacramento deep water channel; Sommer and Mejia 2013; personal observation). Although fine sediment and silt are common substrates in the delta (Keller 2009), it is unlikely that these serve as preferred spawning habitat, as such substrates may restrict oxygen and suffocate eggs (Wyatt et al. 2010). Additionally, while emergent vegetation, cobble, and dead wood can also be found inshore in the delta (personal observation), these did not elicit strong spawning responses in the present study.

In addition to our findings regarding substrate, we also found that $H$. transpacificus deposited more eggs in higher water velocity conditions. However, interpreting these results in relationship to existing conditions in the delta is difficult, as water velocity conditions depend on or are interrelated to many complex, natural processes, including tidal changes and water turbidity (Sommer and Mejia 2013). Thus, H. transpacificus spawning habitat selection in nature is likely to be more complex than the isolated factors tested in this study.

While the results of our study provide a strong foundation on which to base more in-depth research, there are a few limitations to our study that must be considered in conjunction with these results. First, due to the large size of experimental tanks, our study had a limited number of replicates $(N=2$ for experiment $1, N=4$ for experiment 2). Future experiments including a greater number of replicates will be crucial to providing a larger scope of inference for these results. Second, our study did not account for the possible accumulation of eggs on substrates or within certain areas of the tanks that were not the result of explicit choice. For example, the velocity gradient within tanks allowed for the possibility that eggs spawned at lower velocities could be redistributed to higher velocities. However, several points lead us to believe that our results are the product of true spawning response rather than an egg accumulation or locational effect. We counted only eggs that were attached to substrates, excluding any loose eggs that may have been redistributed. Additionally, our results were consistent between experiments, in which substrate placement was offset between velocities and randomized between velocities and within each tank. Our results were also consistent between velocities in experiment 2 . Within the lower $(8.7 \mathrm{~cm} / \mathrm{s})$ velocity tested in experiment 2, egg deposition on pebble and sand was significantly greater than that on all other substrates (post hoc Tukey's multiple comparison test, $P<0.05$ ), but not significantly different from one another (Tukey's, $P>0.05$ ). It is unlikely that egg accumulation alone explains the consistent choice for sand and pebble between velocities and experiments. Furthermore, there was a huge disparity between the number of eggs found on pebble and sand at higher velocities and that found on all other substrate-velocity combinations, which we believe indicates a targeted spawning response. Nevertheless, smaller-scale substrate and velocity choice experiments that include video or direct observation of spawning behavior and randomize substrate and velocity locations would better address these issues.

Despite these limitations, our findings suggest that H. transpacificus may select spawning habitats similar to that of other smelt species. For example, surf smelt Hypomesus pretiosus spawn on beaches in Puget Sound, WA under intertidal conditions on a sand and gravel mix of mostly 1-7 mm sediment size (Penttila 2007). For these smelt, additional environmental factors such as fetch, solar radiation, and beach temperature predict egg abundance (Quinn et al. 2012). In Japan, $H$. pretiosus spawning has been observed on sandy beaches where eggs adhere to sediment grain sizes of about 0.5-2 mm (Hirose and Kawaguchi 1998a, b). In addition, rainbow smelt Osmerus mordax populations in Massachusetts spawn in streams with gravel, pebble, and cobble substrates and water velocities averaging $39 \mathrm{~cm} / \mathrm{s}$ (range = $10-90 \mathrm{~cm} / \mathrm{s}$; Chase 2006). 
This study is the first attempt to characterize spawning microhabitat selection in $H$. transpacificus, as far as we are aware. Our finding that $H$. transpacificus demonstrates differential spawning responses to specific substrates and water velocities under laboratory conditions provides valuable insight into the potential natural habitats that may be used for spawning and ultimately influence reproductive success. This information is particularly important in the case of endangered H. transpacificus, as it can help guide habitat restoration efforts, such as those outlined by the Delta Smelt Resiliency Strategy (California Natural Resources Agency 2016).

Acknowledgments We thank B. Baskerville Bridges and staff (United States Bureau of Reclamation) for their enthusiastic help with fish collection. We also thank L. Sutphin and Fish Conservation and Culture Laboratory staff for their help in completing the experiments, M. Adams, R. Wong, F. Putri, Dr. A. Horst, and Dr. A. Eads for their statistical advice, and Dr. S. Doroshov and Dr. S. Teh for their guidance and support.

Funding Information This study was supported by the Interagency Ecological Program (project \# 2002-049) and California Department of Water Resources (project \#460000 and 460009396).

\section{Compliance with Ethical Standards}

Animal work was conducted in compliance with Institutional Animal Care and Use Committee at University of California, Davis.

Open Access This article is distributed under the terms of the Creative Commons Attribution 4.0 International License (http:// creativecommons.org/licenses/by/4.0/), which permits unrestricted use, distribution, and reproduction in any medium, provided you give appropriate credit to the original author(s) and the source, provide a link to the Creative Commons license, and indicate if changes were made.

\section{References}

Bennett, W.A. 2005. Critical assessment of the delta smelt population in the San Francisco Estuary, California. San Francisco Estuary and Watershed Science, 3(2). Retrieved from https:/escholarship.org/ uc/item/0725n5vk. Accessed 23 Nov 2019.

California Natural Resources Agency. 2016. Delta smelt resiliency strategy.http://resources.ca.gov/docs/Delta-Smelt-ResiliencyStrategy-FINAL070816.pdf. Accessed 10 Oct 2019.

Chase, B.C. 2006. Rainbow smelt (Osmerus mordax) spawning habitat on the Gulf of Maine coast of Massachusetts. Massachusetts division of marine fisheries technical report TR-30. Massachusetts Division of Marine Fisheries. Retrieved from https://www.mass. gov/files/2017-08/tr30-smelt-spawning-habitat.pdf. Accessed 23 Nov 2019.

Department of Fish and Game. 2011. Special Animals (898 taxa). California Natural Diversity Database. Retrieved from http:// www.trpa.org/documents/rseis/3.10\%20Biological\%20Resources/ 3.10_CDFG\%202011_SpecialAnimals.pdf. Accessed 24 Nov 2019.
Hirose, T., and K. Kawaguchi. 1998a. Sediment size composition as an important factor in the selection of spawning site by the Japanese surf smelt Hypomesus japonicus. Fisheries Science 64: 995-996.

Hirose, T., and K. Kawaguchi. 1998b. Spawning ecology of Japanese sand smelt, Hypomesus pretiosus japonicus (Osmeridae), in Otsuchi Bay, northeastern Japan. Environmental Biology of Fishes 52: 213-223.

Interagency Ecological Program. 2015. An updated conceptual model of delta smelt biology: our evolving understanding of an estuarine fish. Technical Report 90. Retrieved from https://www.waterboards.ca. gov/waterrights/water_issues/programs/bay_delta/california waterfix/exhibits/docs/petitioners_exhibit/dwr/part2/DWR-1089\% 20IEP MAST Team 2015 Delta Smelt MAST Synthesis Report_January\%202015.pdf. Accessed 23 Nov 2019.

Keller, B.R. 2009. Literature review of unconsolidated sediment in San Francisco Bay and nearby Pacific Ocean coast. San Francisco Estuary and Watershed Science, 7(1). Retrieved from https:// escholarship.org/uc/item/5rh9t1jj. Accessed 23 Nov 2019.

Lindberg, J.C., G. Tigan, L. Ellison, T. Rettinghouse, M.M. Nagel, and K.M. Fisch. 2013. Aquaculture methods for a genetically managed population of endangered delta smelt. North American Journal of Aquaculture 75: 186-196.

Moyle, P.B., L.R. Brown, J.R. Durand, and J.A. Hobbs. 2016. Delta smelt: life history and decline of a once-abundant species in the San Francisco Estuary. San Francisco Estuary and Watershed Science, 14(2). Retrieved from https://escholarship.org/uc/item/ 09k9f76s. Accessed 23 Nov 2019.

Moyle, P.B., J.A. Hobbs, and J.R. Durand. 2018. Delta smelt and water politics in California. Fisheries 43: 42-50.

Penttila, D. 2007. Marine forage fishes in Puget Sound. Puget Sound Nearshore partnership technical report 2007-03. Washington Department of Fish and Wildlife. Retrieved from http://www. pugetsoundnearshore.org/technical papers/marine fish.pdf . Accessed 23 Nov 2019.

Quinn, T., K. Krueger, K. Pierce, D. Penttila, K. Perry, T. Hicks, and D. Lowry. 2012. Patterns of surf smelt, Hypomesus pretiosus, intertidal spawning habitat use in Puget Sound, Washington state. Estuaries and Coasts 35: 1214-1228.

R Core Team. 2018. R: A language and environment for statistical computing. Vienna, Austria: R Foundation for Statistical Computing.

Radtke, L.D. 1966. Distribution of smelt, juvenile sturgeon, and starry flounder in the Sacramento-San Joaquin Delta with observations on food of sturgeon. In Ecological Studies of the Sacramento-SanJoaquin Delta. Part II: Fishes of the Delta, ed. J.L. Turner and D.W. Kelley, California Department of Fish and Game, Fish Bulletin 136: 115-129.

RStudio Team. 2016. RStudio: Integrated development environment. Boston, MA: RStudio, Inc..

Sommer, T., and F. Mejia. 2013. A place to call home: a synthesis of delta smelt habitat in the upper San Francisco Estuary. San Francisco Estuary and Watershed Science, 11(2). Retrieved from https:// escholarship.org/uc/item/32c8t244. Accessed 23 Nov 2019.

Sommer, T., F. Mejia, M. Nobriga, and L. Grimaldo. 2011. The spawning migration of delta smelt in the upper San Francisco Estuary. San Francisco Estuary and Watershed Science, 9(2). Retrieved from https://escholarship.org/uc/item/86m0g5sz . Accessed November 23, 2019.

Swanson, C.A., P.S. Young, and J.J. Cech Jr. 1998. Swimming performance of delta smelt: maximum performance, and behavioral and kinematic limitations on swimming at submaximal velocities. Journal of Experimental Biology 201: 333-345.

U.S. Fish and Wildlife Service. 1993. Endangered and threatened wildlife and plants: determination of threatened status of the delta smelt. Federal Register 58: 12854-12864. 
Wang, J.C.S. 2010. Fishes of the Sacramento-San Joaquin estuary and adjacent waters, California: a guide to the early life histories. Interagency Ecological Program Technical Report No. 9. U.S. Department of the Interior. Bureau of Reclamation. Mid-Pacific Region. Retrieved from https://www.usbr.gov/mp/TFFIP/docs/ tracy-reports/other-ttr-2010-no9-fishes-sac-sj-estuary.pdf. Accessed 23 Nov 2019.
Wyatt, L.H., A.L. Baker, and D.L. Berlinsky. 2010. Effects of sedimentation and periphyton communities on embryonic rainbow smelt, Osmerus mordax. Aquatic Sciences 72: 361-369.

Zuur, A.F., E.N. Ieno, N.J. Walker, A.A. Saveliev, and G.M. Smith. 2009. Mixed effects models and extensions in ecology with R. New York: Springer. 